\title{
Mesenchymal stem cells accelerated growth and metastasis of neuroblastoma and preferentially homed towards both primary and metastatic loci in orthotopic neuroblastoma model
}

Jiao-Le YU

Beijing Children's Hospital

Shing CHAN

University of Hong Kong

Marcus Kwong-Lam Fung

The University of Hong Kong

Godfrey Chi Fung Chan ( $\nabla$ gcfchan@hku.hk)

University of Hong Kong

Research article

Keywords: mesenchymal stem cells, neuroblastoma, growth, metastasis, homing

Posted Date: October 21st, 2020

DOl: https://doi.org/10.21203/rs.3.rs-21935/v2

License: (c) (i) This work is licensed under a Creative Commons Attribution 4.0 International License.

Read Full License

Version of Record: A version of this preprint was published at BMC Cancer on April 10th, 2021. See the published version at https://doi.org/10.1186/s12885-021-08090-2. 


\section{Abstract}

Background: Majority of neuroblastoma patients develop metastatic disease at diagnosis and their prognosis is poor with current therapeutic approach. Major challenges are how to tackle the mechanisms responsible for tumorigenesis and metastasis. Human mesenchymal stem cells (hMSCs) may be actively involved in the constitution of cancer microenvironment.

Methods: In our study, an orthotopic neuroblastoma murine model was utilized to mimic the actual scenario. Human neuroblastoma cell line SK-N-LP was transfected with luciferase gene, which were inoculated with/without MSCs into the adrenal area of SCID-beige mice. The growth and metastasis of neuroblastoma was observed by using Xenogen IVIS 100 in vivo imaging and evaluating gross tumors ex vivo. The homing of MSCs towards tumor was analyzed by tracing fluorescence signal tagged on MSCs using CRI Maestro ${ }^{\text {TM }}$ imaging system.

Results: hMSCs mixed with neuroblastoma cells significantly accelerated tumor growth and metastasis of neuroblastoma in vivo. hMSCs could be recruited by primary tumor and also become part of the tumor microenvironment in the metastatic lesion. The metastatic potential was significantly reduced when hMSCs were pre-treated with stromal cell derived factor-1 (SDF-1) blocker, AMD3100, suggesting that the SDF-1/CXCR4 axis was a prime mover in the metastatic process.

Conclusions: MSCs accelerated and facilitated tumor formation, growth and metastasis. Furthermore, the homing propensity of MSCs towards both primary tumor and metastatic loci can also provide new therapeutic insights in utilizing bio-engineered MSCs as vehicles for targeted anti-cancer therapy against advanced cancers.

\section{Background}

Neuroblastoma is the most common extra-cranial solid neoplasm in children which accounting for 7-10\% of all pediatric tumors. It is also the most common solid malignancy in children less than one year of age. The incidence of neuroblastoma is 1 in 7000 live births and $96 \%$ of patients are younger than 10 years old [1]. Except a few of those who undergo spontaneous regression during the infancy period, around $65 \%$ of patients have metastatic disease at diagnosis, and they are sensitive to chemotherapy but tend to recur. Despite multimodality therapeutic approaches, most of them have either refractory disease or relapse and the outcome of high-risk neuroblastoma is far from satisfactory even with current therapeutic approach $[2,3]$. How to improve the prognosis of aggressive neuroblastoma remains a major challenge.

The current issue is how to tackle the underlying mechanisms responsible for tumorigenesis and metastasis. The growth and progression of cancer cells require the support from the surrounding microenvironment. Mesenchymal stem cells (or mesenchymal stromal cells, MSCs) have been postulated to be actively involved in the constitution of cancer microenvironment and are responsible for tumorigenesis, metastasis and immune evasion. MSCs are multipotent somatic stem cells. The capability of multi-lineage differentiation and distinct immunomodulatory properties propel MSCs as one of the 
favorite therapeutic cells of choice particularly for tissue regeneration. Paradoxically, such capacities also facilitate tumor cell survival.

The relationship between MSCs and cancer has been considered to be a dual and interactive balance. This fact is reflected by two following evidences. On one hand, MSCs can preferentially traffic towards tumor and thus take part in tumor growth, vice versa, cancer cells can be selectively attracted by MSCs which are mainly resided in bone marrow leading to the bone metastasis; and more importantly, on the other hand, MSCs could exert "beneficial" pro-tumorgenic influence on tumor growth and metastasis. The potential mechanisms underlying "cancer-friendly" effects of MSCs may involve: (1) constructing cancer microenvironment by differentiating into cancer-associated cellular components including fibroblast, pericytes and other connective tissues; (2) enhancing neovascularization through secreting neurogenic and angiogenic factors (e.g. vascular endothelial growth factor); (3) producing growth factors promoting cancer growth; (4) releasing soluble factors to enhance distant metastasis particularly bone metastasis and increasing the survival of cancer cells; (5) regulating the immune system to initiate cancer development [4-7]. Although majority of studies support MSCs can accelerate the growth and progression of tumor [5, 8-10], however, anti-cancer effects were also observed in other studies [11-13]. So far, the impact of MSCs in cancer microenvironment remains controversial. It has been indicated that the survival of neuroblastoma could be supported by MSCs in some in vitro studies [14,15]. Our previous study has demonstrated that MSCs could enhance the metastasis of neuroblastoma via stromal cell derived factor1 (SDF-1) signaling pathway in vitro [16]. However, opposite results was also reported [17]. Underlying mechanisms attributed to the contrary research results are potentially depending on the particular research design, different in vitro culture conditions and particularly the tumor model. It has been argued whether the current tumor model exactly reflects the natural microenvironment since majority of studies established animal tumor model by subcutaneous transplantation. A suitable orthotopic preclinical model is required to mimic the real scenario. We therefore aim to understand this dynamic relationship by performing cellular mixing experiments and co-implant MSCs with neuroblastoma cells in vivo.

In this study, an orthotopic murine model was adopted to investigate the impact of MSCs on tumorigenesis and metastasis of neuroblastoma. Homing property and the underlying mechanisms was further explored.

\section{Methods}

\section{Materials and reagents}

AMD3100, a specific antagonist of Stromal cell-derived factor-1a (SDF-1a)'s receptor CXCR4, was purchased from Sigma-Aldrich (St. Louis, MO, USA). Lipofectamine ${ }^{\circledR} 2000$ was from Invitrogen (Carlsbad, CA, USA). Lipophilic fluorescence dye-CM Dil was from Molecular Probes (Carlsbad, CA, USA).

\section{Cell culture}


The bone marrow human MSCs (hMSCs) were isolated from healthy bone marrow transplantation donors by density-gradient centrifugation with Ficoll-Hypaque (GE Healthcare, Little Chalfont, UK). Written informed consent was obtained under the approval of the Combined Internal Review Board (Ethical Committee) of the University of Hong Kong and The Hong Kong West Cluster of Hospital Authority. The immunophenotype and differentiation characteristics of MSCs were clarified by surface marker definition and differentiation assays. The homogenous hTertMSCs, an immortalized hMSCs cell line with human telomerase reverse transcriptase gene inserted, was a gift from Prof. D. Campana (St Jude Children's Research Hospital, Memphis, TN, USA) [18]. All hMSCs were cultured in vitro with Dulbecco's Modified Eagles Medium-low glucose (DMEM-LG; GIBCO, Invitrogen, Carlsbad, CA, USA) supplemented with 10\% fetal bovine serum (FBS), $100 \mathrm{U} / \mathrm{mL}$ penicillin, $100 \mathrm{mg} / \mathrm{mL}$ streptomycin and $2 \mathrm{mM}$ L-Glutamine.

Human neuroblastoma cell line SK-N-LP (a gift from Prof. NK Cheung, Memorial Sloan-Kettering Cancer Centre, NY, USA) was cultured with Dulbecco's Modified Eagles Medium-high glucose (DMEM-HG, Invitrogen, Carlsbad, CA, USA) at $37^{\circ} \mathrm{C}$ supplemented with $10 \% \mathrm{FBS}, 100 \mathrm{U} / \mathrm{mL}$ penicillin, $100 \mathrm{mg} / \mathrm{mL}$ streptomycin and $2 \mathrm{mM} \mathrm{L-Glutamine.}$

\section{Cell labeling}

hMSCs were pre-labeled with the lipophilic fluorescence dye-CM Dil (Molecular Probes, Carlsbad, CA, USA) before in vivo transplantation. In brief, cells were washed and incubated with CM-Dil at concentration of $5 \mu \mathrm{l} / \mathrm{mL}$ for 20 minutes at $37^{\circ} \mathrm{C}$ and then washed three times with normal growth medium according to the instructions of the manufacturer. The concentration and incubation period were optimized by series of tests. The labeling efficiency was detected to be more than $99 \%$ without cytotoxicity and the strong fluorescence signal has been proven to be persistent for more than one month.

\section{Cell transfection and culture of bioluminescent human neuroblastoma cell line}

Human neuroblastoma cell line SK-N-LP were transfected with plasmid expressing luciferase (a kind gift from Prof. Kwan Man) using Lipofectamine ${ }^{\circledR} 2000$ (Invitrogen, Carlsbad, CA, USA) following the manufacturer's instructions. In brief, cells were cultured in 6-well plate and allowed to grow until they were $70-80 \%$ confluent. Plasmid DNA was diluted with DMEM without FBS and mixed with prepared Lipofectamine ${ }^{\circledR} 2000$ solution. The mixture was added into cells and cultured at $37^{\circ} \mathrm{C}$ overnight. Stable cell line was obtained by neomycin (G418) selection. The bioluminescence of luciferase gene-transfected cells was confirmed under Xenogen IVIS 100 imaging system. Luciferase gene-transfected SK-N-LP cells were culture with DMEM-HG supplemented with $10 \% \mathrm{FBS}$ at $37^{\circ} \mathrm{C}$ and sub-cultured when growing to 70 $80 \%$ confluence.

\section{Orthotopic neuroblastoma modeland xenografts of human luciferase-SK-N-LP cells}

This in vivo project obtained the approval of Hong Kong Department of Health and Committee on the Use of Live Animals in Teaching and Research (CULATR), The University of Hong Kong. All procedures and animal care were under the surveillance of the committee. 6-week severe combined immunodeficiency 
(SCID)-beige mice $(18.73 \pm 0.76 \mathrm{~g})$ were obtained from the Laboratory Animal Unit, The University of Hong Kong and were housed at specific pathogen-free facility with temperature of $22 \pm 1^{\circ} \mathrm{C}$, humidity of $55 \pm 5 \%$ and bred with autoclaved food and water ad libitum. Animals were monitored twice daily.

Luciferase-SK-N-LP cells were trypsinized from the culture flasks and prepared into single cell suspension. Cell viability was analyzed to be more than $99 \%$ using trypan blue exclusion assay. The single cell suspension at a concentration of $2 \times 10^{7} / \mathrm{mL}$ and $0.2 \times 10^{6}$ cells diluted with $10 \mu 150 \%$ matrigel (BD Bioscience, Bedford, MA, USA) were prepared in equal volume and maintained on ice. The mixture of SK-N-LP and hMSCs was prepared by mixing SK-N-LP and hMSCs in ratio of 2:1. The final injected number of SK-N-LP cells was $0.2 \times 10^{6}$.

Animals were anesthetized by intra-peritoneal injection of $100 \mathrm{mg} / \mathrm{kg}$ pentobarbital. After the disinfection with alcohol and betadine, an incision was made vertically in the abdomen of anesthetized SCID-beige mice. Left kidney was exposed gently and $20 \mu$ l of cell mixture was slowly injected into the fat pad of the adrenal gland adjacent to the upper pole of left kidney. The organs were carefully rearranged back and the incision was closed. The whole surgery was ensured to be aseptic avoiding infection. Mice were monitored until regaining consciousness. During the first 3 days post-surgery, the mice were given meloxicam in drinking water to minimize the pain at the dose of $0.3 \mathrm{mg} / \mathrm{kg}$.

Following the intraperitoneal injection with D-luciferin (Gold Biotechnology, St Louis, MO, USA), the bioluminescence of transplanted cells of SK-N-LP group $(n=4)$ and hMSCs co-transplantation group $(n=4)$ was compared by Xenogen IVIS 100 in vivo imaging which was used to evaluate the in vivo initiation and progression of neuroblastoma. Signal intensity of regions of interest was analyzed by Living Image ${ }^{\circledR}$ Software (Xenogen, corporation Alameda, CA). Study designed for exploring the role of hMSCs in the growth and metastasis of neuroblastoma was shown in Supplementary Figure 1.

\section{Treatment and transplantation of hMSCs}

CM Dil pre-labeled hMSCs $\left(1 \times 10^{6}\right)$ were cultured with PBS (Control group, $\mathrm{n}=4$ ) or specific CXCR4 antagonist AMD3100 $(10 \mu \mathrm{g} / \mathrm{mL})$ in suspension for 1 hour, respectively. Then they were intravenously injected into mice with implanted neuroblastoma 7 weeks post-surgery via tail vein $(n=4)$. Before injection, the staining efficiency and cell viability were evaluated. Experimental design for exploring the tumor tropism property of hMSCs towards primary tumor and metastatic loci was illustrated in

\section{Supplementary Figure 1.}

\section{Tumor volume evaluation}

Three dimensions of isolated tumors were measured using a digital caliper and the volume was calculated according to the following formula. Tumor volume=Length $\times$ Wideth $\times$ Height $\times 1 / 2$.

\section{Tumor metastasis loci detection}


Mice were sacrificed by an overdose of pentobarbital. Organs including brain, lung, heart, liver, spleen, gut and bone were harvested immediately and washed twice with PBS. The metastatic loci of neuroblastoma were detected using Xenogen IVIS 100 imaging.

\section{Evaluation of hMSCs trafficking in vivo}

The trafficking of hMSCs in vivo was traced using CRI Maestro ${ }^{\mathrm{TM}}$ imaging system by detecting the fluorescence signal of CM-Dil pre-labeled hMSCs in freshly harvested tumors and organs.

\section{Statistical analysis}

Comparison between means from different groups was analyzed using unpaired 2-tailed Student $t$ test for tumor volume. The difference was considered as statistically significant only when $P<0.05$. The statistical analysis and data graphs were conducted by GraphPad Prism 5 (GraphPad Software Inc, San Diego, CA, USA).

\section{Results}

\section{Characteristics of orthotopic neuroblastoma murine model}

Transplantation of human SK-N-LP cells into SCID-beige mice was demonstrated as being capable of generating stable orthotopic neuroblastoma which was validated by both in vivo imaging of detecting the bioluminescent signals tagged at SK-N-LP cells and by evaluating the gross tumors harvested from mice. The pronounced progression of tumor growth after the xenogeneic transplantation of human neuroblastoma cell line was shown in Figure 1. Notable increase of tumor size was detected from Day 28 (4/4), Day 49 to Day 56 (3/4) post-inoculation (Fig. 1A). Correlatively, rising intensity of photon emission was observed using Xenogen IVIS in vivo imaging (Fig. 1B).

In accordance with the clinical manifestations, this neuroblastoma model demonstrated the characteristics of metastasis in multiple organs. All mice developed metastatic disease in multiple organs. Bone, brain, lung, liver, and gastrointestinal tract were invaded. It was observed that liver, lung, gut and bone were the major organs invaded in almost all the mice (Fig. 1C(i)). In addition, our mice also exhibited the variable clinical behaviors of metastasis observed in humans. Some mice, however, exhibited special symptoms. In some mice, the major organs involved were bone (Fig. 1C(ii)) and spinal cord (Fig. 1D) rather than liver and lung. And these mice developed severe paralysis of lower limbs and were clinically unable to crawl. One of mice from each group was received euthanasia by an overdose of pentobarbital before Day 56. Gastrointestinal tract and kidney encompassed by tumor was commonly seen phenomenon in all the mice, and the adhesion of tumor to liver or lower spine appeared in some mice suggesting this neuroblastoma cell line SK-N-LP is highly invasive and can progress in diverse directions (Fig. 1D). 
As early as the Day 28 post-transplantation, clear bioluminescent signals were detectable using Xenogen IVIS 100 imaging indicating the early formation of tumor in vivo. Compared to SK-N-LP only transplantation group, the bioluminescent signal was notably stronger in the group receiving the cotransplantation of hMSCs and SK-N-LP suggesting hMSCs may accelerate the in vivo initiation of neuroblastoma (Fig. 2A(i)). The difference in neuroblastoma progression was even more pronounced for mice with 56 days of tumor implantation (Fig. 2A(ii)) and was further confirmed by the calculation of harvested gross tumor volume. The volume and size of tumors harvested from co-transplantation group was significantly higher than these isolated from SK-N-LP alone group (Fig. 2B, 2C). Above results supported that hMSCs promoted the early initiation and progression of neuroblastoma in vivo.

\section{hMSCs promoted the metastasis of neuroblastoma}

Since hMSCs indicated favorable impact on tumor growth, we further evaluated whether they could promote metastasis as well. At Day56 post-inoculation, bioluminescent signals were analyzed using IVIS 100 imaging system. In co-transplantation group, all organs (lung, liver, gastrointestinal tract, brain and bone) gave the bioluminescence, whereas, not all mice in SK-N-LP group developed metastatic disease in all observed organs. The bioluminescence was detectable in restricted organs with weaker intensity (Fig. 2D). Based on this observation, we postulated that hMSCs could potentially enhance local or distant metastasis of neuroblastoma but the involved sites of metastasis might happen by chance.

\section{Recruitment of hMSCs towards primary tumor was in a CXCR4-dependent manner}

CM-Dil-labeled hMSCs pre-treated with or without AMD3100 were injected into mice with neuroblastoma, the fluorescence signal was detected using in vivo imaging. We observed that the fluorescence signal of CM-Dil could be detected at primary tumor site indicating hMSCs were attracted by neuroblastoma; however, the signal intensity was significantly reduced when hMSCs were pre-treated with AMD3100 suggesting that this trafficking process was abolished by AMD3100 (Fig. 3). These observations revealed that hMSCs could preferentially home towards primary tumor and more importantly, in a CXCR4dependent manner.

\section{hMSCs could be recruited by the metastatic loci of neuroblastoma}

In addition to the homing towards primary tumor, CM-Dil labeled hMSCs could also be detected at metastasis loci of neuroblastoma as shown in Figure 4; however, the fluorescence signal of hMSCs could not be detected in non-invaded liver (Fig. 4B) and lung using in vivo imaging (Fig. 4E). Such findings indicated that hMSCs could be selectively recruited by the metastatic loci of neuroblastoma. In contrary to the potent suppressive influence on the trafficking towards the primary tumor site, AMD3100 treatment could not completely inhibit the recruitment of MSCs to the metastatic loci of neuroblastoma (Fig. 5)

\section{Discussion}


In our present study, we utilized an orthotopic murine model of neuroblastoma to mimick the actual neuroblastoma condition in vivo. Adrenal gland is the most common primary site of neuroblastoma and implanting xenografts of human neuroblastoma cells into the adrenal gland area provide a more relevant microenvironment setting compared to the subcutaneously implanted in vivo tumor model. The orthotopic xenograft offers a more ideal in vivo condition to mimic the relationship between cancer cells and the microenvironment. This model also allows us to evaluate the metastasis potential of neuroblastoma cells in vivo. In our model, bioluminescence gene luciferase was used to label transplanted tumor cells, which can be utilized to monitor the early growth of tumor in vivo noninvasively. This model is free of apparent auto-fluorescence background since luciferase cannot be produced naturally by mice. Thus, this orthotopic model provides a better platform for investigating the underlying tumorigenic pathophysiology, and furthermore, for evaluating the efficacy of novel anti-cancer therapies.

We further explored the effects of MSCs on the initiation and progression of human neuroblastoma. Until now, the exact impact of MSCs on tumor growth and progression in vivo is still controversial. The favorable effects of MSCs have been reported in the tumorigenesis of ovarian cancer [19], colorectal carcinoma [20], breast cancer [8] and pancreatic cancer [9]. The potential underlying mechanisms include the supportive effects of MSCs on cancer growth and metastasis. MSCs could enhance tumor growth via constituting the cancer microenvironment, enhancing neovascularization, producing growth factors and exerting immunosuppressive effects. In addition, MSCs enhance cancer metastasis through releasing soluble factors such as chemokine SDF-1, IL-6 and CCL5. They impact on cancer metastasis through releasing soluble factors such as chemokine SDF-1, IL-6 and CCL5 [4-6]. Moreover, MSCs protect cancer cell survival from cytotoxicity of anti-cancer reagents [21]. However, MSCs also exerted negative effects on the growth of colon carcinoma [22], Kaposi's carcinoma [23], glioma [12] and hepatoma model [11]. Conflict findings were reported even in same type of cancer or study including neuroblastoma $[14,15,17$, 24-27]. To date, the exact reasons underlying these controversial effects remain largely unknown. It is potentially related to the specific histological types of cancer, particular experimental model and research design, different in vitro culture conditions and the dosage of cell inoculation. Furthermore, such effects could also be closely related with tumor-specific background and in certain scenarios even be cell-line specific. Majority of studies observed the supportive effects of co-transplanted MSCs on cancers using excessive number of MSCs than cancer cells or at least an equal number. Specially, my study validated that lower dosage of MSCs co-injection using $0.5^{\prime} 10^{5} \mathrm{hMSCs}$ and $1^{\prime} 10^{5}$ neuroblastoma cells (hMSCs:NB cells=1:2) was enough to generate the promoting effects on tumor growth and metastasis. However, much lower dosage of hMSCs $\left(10^{2}\right.$ hMSCs to $10^{4}$ cancer cells, i.e. hMSCs:cancer cells $\left.=1: 100\right)$ was found to induce tumor rejection [28].

The data from current research focusing on the interaction between MSCs and neuroblastoma is very limited. It was revealed that MSCs could protect neuroblastoma from oxidative stress in vitro [29]. IL-6 produced by MSCs was reported to participate in promoting survival of neuroblastoma cells and the bone metastasis [30]. In addition, SDF-1/CXCR4 axis plays a pivotal role in growth, progression and metastasis 
modulation in diverse kinds of cancers including head and neck cancer, pancreatic cancer and lung cancer. It was suggested that SDF-1/CXCR4 axis could promote the dissemination of cancer cells towards sites highly secreting SDF-1 through binding to the cognate receptor CXCR4 expressed on cancer cells including neuroblastoma [31-33]. Our previous in vitro study demonstrated that MSCs could benefit the metastasis of neuroblastoma via the secretion of SDF-1 [16]. It was also reported that MSCs secretome could modulate CXCR4 expression and invasion to the bone marrow of neuroblastoma in vitro [34]. Despite the above progress, the exact role of MSCs in neuroblastoma development has yet to be defined and majority of data require further validation by in vivo experiments. In this study, using in vivo model, we demonstrated that hMSCs indeed exerted tumorigenic effects on neuroblastoma in vivo. In early period of post-inoculation, mice co-transplanted with hMSCs and SK-N-LP showed stronger tumor signals compared to the mice injected with SK-N-LP alone. Such phenomenon was further verified by evaluation of gross tumor volume. The facilitative effect of hMSCs on neuroblastoma's metastasis was also studied. We observed that compared to SK-N-LP group, MSCs co-transplantation may accelerate the metastasis since all mice in this group developed metastasis in all organs studied and had stronger bioluminescent signals.

Based on the above results, we further explored whether hMSCs could be a therapeutic target to eradicate tumors from the microenvironment shelter. To achieve this goal, we investigated the trafficking of hMSCs in mice bearing neuroblastoma and investigated the potential modifier involved in this whole process. It has been extensively reported that SDF-1/CXCR4 axis is actively involved in the homing of MSCs to injured tissues and thereafter exert biological immunomodulatory and regeneration effects. MSCs were also found to have the propensity of being guided towards tumors. However, unlike the advanced understanding of MSCs trafficking to injured tissues, the mechanism responsible for homing of MSCs towards tumors is just starting to be unfolded and it has not been adequately explored especially in the setting of neuroblastoma. Whether SDF-1/CXCR4 axis is a vital modifier in MSCs homing towards neuroblastoma, like it is described in the trafficking towards injured tissues is still uncertain. We found that hMSCs could preferentially migrate to neuroblastoma. Importantly, we also demonstrated that such trafficking was in a CXCR4-dependent manner. Pretreatment with AMD3100, the specific antagonist of CXCR4, significantly abolished the homing of hMSCs towards primary tumor. The strong evidence supporting this concept came from the striking phenomenon that no hMSCs signal could be detected in liver or lung without metastatic disease. Moreover, supplementing the early studies, we observed that systemically infused hMSCs could also be attracted by the metastatic loci other than the primary tumor.

To the best of our knowledge, few studies have demonstrated the preferential homing of MSCs towards metastatic loci. One study reported intravenously injected MSCs could be guided towards primary tumor and lung metastatic sites. However, they established the tumor model using subcutaneous inoculation and the lung metastasis was induced separately through intravenous injection of tumor cells. After systemically infusing the MSCs 4 days post-injection of cancer cells, they indicated higher signal intensity and longer retention of MSCs at lung than normal control and thus proposed the specific recruitment of MSCs by metastatic lesions [35]. This can be due to trapping of cancer cells in the lung tissue via the "first-pass effect" during venous return to the right heart and then the lung. In our study, an 
orthotopic tumor model was established, and other than lung invasion, multiple metastatic diseases were triggered naturally by generated primary tumor. Using this clinically relevant model, we provided convincing evidence for the tumor tropism of MSCs. Firstly it was demonstrated that intravenously injected hMSCs could preferentially home towards both the primary tumor site and the multiple metastatic loci. And more strikingly, through labeling the hMSCs and tumor cells, we can directly observe the preferential migration of hMSCs to organs invaded, whereas, no hMSCs could be detected in normal tissues. Moreover, such finding was further supported by the observation that higher intensity of hMSCs signal was observed in organs with higher degree of invasion indicating indeed hMSCs could only be recruited by tumor cells at both primary site or metastatic loci and such homing was correlated with the invasive tumor cell number. This property of tropism could provide a promising cue for targeting the microenvironment in high risk metastatic neuroblastoma.

Interestingly, albeit significant inhibitory effects on hMSCs trafficking towards primary tumor, AMD3100 pretreatment failed to show similar impact on the recruitment of hMSCs by the metastasis loci. The mechanism underlying this paradoxical phenomenon warrants further detailed investigations. We hypothesize several possible mechanisms underlying this phenomenon. The first potential reason is the level of SDF-1 was much higher at metastatic loci than primary site [36]. The inhibition of MSCs homing towards metastatic loci may require much higher dosage of AMD3100 than used in blocking the migration of MSCs towards primary site. In support of this, using high-density tissue microarrays, a large cohort study of more than 600 human prostate carcinoma specimens indicated that compared to primary tumor sites, higher SDF-1 was expressed by metastatic lesions [37]. In addition, CXCR7, the other receptor of SDF-1, was reported to express in cancer cells and associated with tumorigenesis and metastasis [36, $38,39]$. In our previous study, both CXCR4 and CXCR7 were found to express in neuroblastoma cell lines. CXCR7 was involved in increasing neuroblastoma migration acting as alternative receptor of SDF-1 in the absence of CXCR4, but not functional in regulating cell migration and adhesion [16]. However, the exact role of CXCR7 in guiding the homing of MSCs towards tumor metastatic loci deserves further study. Finally, the potential variation in microenvironment between primary tumors and metastatic lesions may result in different profiles of released chemokines. The metastatic lesions might trigger different tissue injury signals and involved other non-SDF-1 related pathways.

The limitations of current study could not ascertain the effects of hMSCs on early metastasis of neuroblastoma, which requires further in vivo studies. The sequence in which intravenously infused hMSCs migrate towards primary tumor or metastatic loci remains unknown. Moreover, one notable phenomenon that AMD3100 pre-treatment could not inhibit the homing of hMSCs towards metastatic loci requires further verification. In addition, albeit useful, it was observed that the bioluminescence imaging underestimated the tumor burden at longer time period.

\section{Conclusions}

In conclusion, a stable xenogeneic orthotopic neuroblastoma model could be established using adrenal injection with human neuroblastoma cells and was validated to be able to grow and metastasize to a 
variety of distant sites. Bioluminescence detection could be used to monitor the tumor growth especially during the early initiation phase of tumor in vivo. hMSCs exhibited potent effects on accelerating the potency of tumor formation, growth and metastasis. These results provide the evidence of using MSCs as the promising therapeutic target in the future clinical applications. Furthermore, the preferential homing propensity of MSCs towards both primary tumor and metastatic loci could also bring the new therapeutic insights in utilizing bio-engineered MSCs as vehicles for targeted anti-cancer therapy especially against the advanced diseases (Fig 6).

\section{Abbreviations}

hMSCs: Human mesenchymal stem cells; SDF-1: Stromal cell derived factor-1; CXCR4: C-X-C chemokine receptor4; MSCs: Mesenchymal stem cells; DMEM: Dulbecco's Modified Eagles Medium; FBS: fetal bovine serum; IVIS 100: In vivo imaging system Xenogen IVIS100

\section{Declarations}

We declare no conflict of interest.

\section{Acknowledgements}

We thank our lab-mates Mr Xiang Zheng, Mr Liu Yan (The University of Hong Kong) for their help in my in vivo study.

\section{Funding}

This project was supported in part by the Hotung SK Fund, CRCG grant (grant number: 200811159071, The University of Hong Kong), TTRAS grant, National Science Foundation for Distinguished Young Scholars of China (grant number: 81400074) and Beijing Hospital Authority Youth Programme (QML20161202). The funding bodies have no role in the design of the study; collection, analysis and interpretation of data; and in writing the manuscript.

\section{Availability of data and materials}

The data sets analyzed during the current study are available from the corresponding author, Prof. G.C.F Chan, on reasonable request.

Author's Contributions: J.L.Y. designed the research, performed experiments, analyzed data and wrote the manuscript. S. C participated in operating CRI Maestro ${ }^{T M}$ imaging system. M.K.L.F analyzed data and revised the manuscript. G.C.F.C. designed the research, interpreted data, revised and final approved the manuscript.

\section{Competing interests}


The authors state no conflict of interest.

\section{Consent for publication}

Not applicable.

\section{Ethics approval and consent to participate}

The in vivo project obtained the approval of Hong Kong Department of Health and Committee on the Use of Live Animals in Teaching and Research (CULATR), The University of Hong Kong. MSCs were isolated from healthy bone marrow transplantation donors. Written informed consent was obtained under the approval of the Combined Internal Review Board (Ethical Committee) of the University of Hong Kong and The Hong Kong West Cluster of Hospital Authority.

\section{Author details}

${ }^{1}$ Hematology Oncology Center, Beijing Children's Hospital, Capital Medical University, National Center for Children's Health, Beijing, China; ${ }^{2,3}$ Department of Paediatrics and Adolescent Medicine, Li Ka Shing Faculty of Medicine, The University of Hong Kong, Hong Kong Special Administrative Region, China

\section{References}

1. Matthay, K.K., Maris, J.M., Schleiermacher, G., Nakagawara, A., Mackall, C.L., Diller, L., and Weiss, W.A., Neuroblastoma. Nat Rev Dis Primers. 2016; 2:16078.

2. Ladenstein, R., Potschger, U., Valteau-Couanet, D., Luksch, R., Castel, V., Yaniv, I., Laureys, G., Brock, P., Michon, J.M., Owens, C., Trahair, T., Chan, G.C.F., Ruud, E., Schroeder, H., Beck Popovic, M., Schreier, G., Loibner, H., Ambros, P., Holmes, K., Castellani, M.R., Gaze, M.N., Garaventa, A., Pearson, A.D.J., and Lode, H.N., Interleukin 2 with anti-GD2 antibody ch14.18/CHO (dinutuximab beta) in patients with high-risk neuroblastoma (HR-NBL1/SIOPEN): a multicentre, randomised, phase 3 trial. Lancet Oncol. 2018; 1912:1617-29.

3. Siegel, R.L., Miller, K.D., and Jemal, A., Cancer Statistics, 2017. CA Cancer J Clin. 2017; 671:7-30.

4. Shi, Y., Du, L., Lin, L., and Wang, Y., Tumour-associated mesenchymal stem/stromal cells: emerging therapeutic targets. Nat Rev Drug Discov. 2017; 161:35-52.

5. Ridge, S.M., Sullivan, F.J., and Glynn, S.A., Mesenchymal stem cells: key players in cancer progression. Mol Cancer. 2017; 161:31.

6. Melzer, C., von der Ohe, J., Lehnert, H., Ungefroren, H., and Hass, R., Cancer stem cell niche models and contribution by mesenchymal stroma/stem cells. Mol Cancer. 2017; 161:28.

7. Atiya, H., Frisbie, L., Pressimone, C., and Coffman, L., Mesenchymal Stem Cells in the Tumor Microenvironment. Adv Exp Med Biol. 2020; 1234:31-42.

8. Gonzalez, M.E., Martin, E.E., Anwar, T., Arellano-Garcia, C., Medhora, N., Lama, A., Chen, Y.C., Tanager, K.S., Yoon, E., Kidwell, K.M., Ge, C., Franceschi, R.T., and Kleer, C.G., Mesenchymal Stem Cell-Induced 
DDR2 Mediates Stromal-Breast Cancer Interactions and Metastasis Growth. Cell Rep. 2017; 185:1215-28.

9. Blogowski, W., Bodnarczuk, T., and Starzynska, T., Concise Review: Pancreatic Cancer and Bone Marrow-Derived Stem Cells. Stem Cells Transl Med. 2016; 57:938-45.

10. Yu, J.M., Jun, E.S., Bae, Y.C., and Jung, J.S., Mesenchymal stem cells derived from human adipose tissues favor tumor cell growth in vivo. Stem Cells Dev. 2008; 173:463-73.

11. Qiao, L., Xu, Z., Zhao, T., Zhao, Z., Shi, M., Zhao, R.C., Ye, L., and Zhang, X., Suppression of tumorigenesis by human mesenchymal stem cells in a hepatoma model. Cell Res. 2008; 184:500-7.

12. Ho, I.A., Toh, H.C., Ng, W.H., Teo, Y.L., Guo, C.M., Hui, K.M., and Lam, P.Y., Human bone marrow-derived mesenchymal stem cells suppress human glioma growth through inhibition of angiogenesis. Stem Cells. 2013; 311:146-55.

13. Song, N., Gao, L., Qiu, H., Huang, C., Cheng, H., Zhou, H., Lv, S., Chen, L., and Wang, J., Mouse bone marrow-derived mesenchymal stem cells inhibit leukemia/lymphoma cell proliferation in vitro and in a mouse model of allogeneic bone marrow transplant. Int J Mol Med. 2015; 361:139-49.

14. Pires, A.O., Neves-Carvalho, A., Sousa, N., and Salgado, A.J., The Secretome of Bone Marrow and Wharton Jelly Derived Mesenchymal Stem Cells Induces Differentiation and Neurite Outgrowth in SHSY5Y Cells. Stem Cells Int. 2014; 2014:438352.

15. Curtis, T.M., Hannett, J.M., Harman, R.M., Puoplo, N.A., and Van de Walle, G.R., The secretome of adipose-derived mesenchymal stem cells protects SH-SY5Y cells from arsenic-induced toxicity, independent of a neuron-like differentiation mechanism. Neurotoxicology. 2018; 67:54-64.

16. Ma, M., Ye, J.Y., Deng, R., Dee, C.M., and Chan, G.C., Mesenchymal stromal cells may enhance metastasis of neuroblastoma via SDF-1/CXCR4 and SDF-1/CXCR7 signaling. Cancer Lett. 2011; 3121:1-10.

17. Bianchi, G., Morandi, F., Cilli, M., Daga, A., Bocelli-Tyndall, C., Gambini, C., Pistoia, V., and Raffaghello, L., Close interactions between mesenchymal stem cells and neuroblastoma cell lines lead to tumor growth inhibition. PLoS One. 2012; 710:e48654.

18. Mihara, K., Imai, C., Coustan-Smith, E., Dome, J.S., Dominici, M., Vanin, E., and Campana, D., Development and functional characterization of human bone marrow mesenchymal cells immortalized by enforced expression of telomerase. Br J Haematol. 2003; 1205:846-9.

19. Chu, Y., Tang, H., Guo, Y., Guo, J., Huang, B., Fang, F., Cai, J., and Wang, Z., Adipose-derived mesenchymal stem cells promote cell proliferation and invasion of epithelial ovarian cancer. Exp Cell Res. 2015; 3371:16-27.

20. Nishikawa, G., Kawada, K., Nakagawa, J., Toda, K., Ogawa, R., Inamoto, S., Mizuno, R., Itatani, Y., and Sakai, Y., Bone marrow-derived mesenchymal stem cells promote colorectal cancer progression via CCR5. Cell Death Dis. 2019; 104:264.

21. Fung, K.L., Liang, R.H., and Chan, G.C., Vincristine but not imatinib could suppress mesenchymal niche's support to lymphoid leukemic cells. Leuk Lymphoma. 2010; 513:515-22. 
22. Ohlsson, L.B., Varas, L., Kjellman, C., Edvardsen, K., and Lindvall, M., Mesenchymal progenitor cellmediated inhibition of tumor growth in vivo and in vitro in gelatin matrix. Exp Mol Pathol. 2003; 753:248-55.

23. Khakoo, A.Y., Pati, S., Anderson, S.A., Reid, W., Elshal, M.F., Rovira, II, Nguyen, A.T., Malide, D., Combs, C.A., Hall, G., Zhang, J., Raffeld, M., Rogers, T.B., Stetler-Stevenson, W., Frank, J.A., Reitz, M., and Finkel, T., Human mesenchymal stem cells exert potent antitumorigenic effects in a model of Kaposi's sarcoma. J Exp Med. 2006; 2035:1235-47.

24. Klopp, A.H., Gupta, A., Spaeth, E., Andreeff, M., and Marini, F., 3rd, Concise review: Dissecting a discrepancy in the literature: do mesenchymal stem cells support or suppress tumor growth? Stem Cells. 2011; 291:11-9.

25. Lee, H.Y. and Hong, I.S., Double-edged sword of mesenchymal stem cells: Cancer-promoting versus therapeutic potential. Cancer Sci. 2017; 10810:1939-46.

26. Li, W., Ren, G., Huang, Y., Su, J., Han, Y., Li, J., Chen, X., Cao, K., Chen, Q., Shou, P., Zhang, L., Yuan, Z.R., Roberts, A.I., Shi, S., Le, A.D., and Shi, Y., Mesenchymal stem cells: a double-edged sword in regulating immune responses. Cell Death Differ. 2012; 199:1505-13.

27. Tian, L.L., Yue, W., Zhu, F., Li, S., and Li, W., Human mesenchymal stem cells play a dual role on tumor cell growth in vitro and in vivo. J Cell Physiol. 2011; 2267:1860-7.

28. Djouad, F., Bony, C., Apparailly, F., Louis-Plence, P., Jorgensen, C., and Noel, D., Earlier onset of syngeneic tumors in the presence of mesenchymal stem cells. Transplantation. 2006; 828:1060-6.

29. Lanza, C., Morando, S., Voci, A., Canesi, L., Principato, M.C., Serpero, L.D., Mancardi, G., Uccelli, A., and Vergani, L., Neuroprotective mesenchymal stem cells are endowed with a potent antioxidant effect in vivo. J Neurochem. 2009; 1105:1674-84.

30. Ara, T., Song, L., Shimada, H., Keshelava, N., Russell, H.V., Metelitsa, L.S., Groshen, S.G., Seeger, R.C., and DeClerck, Y.A., Interleukin- 6 in the bone marrow microenvironment promotes the growth and survival of neuroblastoma cells. Cancer Res. 2009; 691:329-37.

31. De-Colle, C., Menegakis, A., Monnich, D., Welz, S., Boeke, S., Sipos, B., Fend, F., Mauz, P.S., Tinhofer, I., Budach, V., Abu Jawad, J., Stuschke, M., Balermpas, P., Rodel, C., Grosu, A.L., Abdollahi, A., Debus, J., Belka, C., Ganswindt, U., Pigorsch, S., Combs, S.E., Lohaus, F., Linge, A., Krause, M., Baumann, M., Zips, D., and Dktk, R.O.G., SDF-1/CXCR4 expression is an independent negative prognostic biomarker in patients with head and neck cancer after primary radiochemotherapy. Radiother Oncol. 2018; 1261:125-31.

32. Sleightholm, R.L., Neilsen, B.K., Li, J., Steele, M.M., Singh, R.K., Hollingsworth, M.A., and Oupicky, D., Emerging roles of the CXCL12/CXCR4 axis in pancreatic cancer progression and therapy. Pharmacol Ther. 2017; 179:158-70.

33. Katsura, M., Shoji, F., Okamoto, T., Shimamatsu, S., Hirai, F., Toyokawa, G., Morodomi, Y., Tagawa, T., Oda, Y., and Maehara, Y., Correlation between CXCR4/CXCR7/CXCL12 chemokine axis expression and prognosis in lymph-node-positive lung cancer patients. Cancer Sci. 2018; 1091:154-65. 
34. Shankar, V., Hori, H., Kihira, K., Lei, Q., Toyoda, H., Iwamoto, S., and Komada, Y., Mesenchymal stromal cell secretome up-regulates $47 \mathrm{kDa}$ CXCR4 expression, and induce invasiveness in neuroblastoma cell lines. PLoS One. 2015; 103:e0120069.

35. Wang, H., Cao, F., De, A., Cao, Y., Contag, C., Gambhir, S.S., Wu, J.C., and Chen, X., Trafficking mesenchymal stem cell engraftment and differentiation in tumor-bearing mice by bioluminescence imaging. Stem Cells. 2009; 277:1548-58.

36. Wang, M., Yang, X., Wei, M., and Wang, Z., The Role of CXCL12 Axis in Lung Metastasis of Colorectal Cancer. J Cancer. 2018; 921:3898-903.

37. Sun, Y.X., Wang, J., Shelburne, C.E., Lopatin, D.E., Chinnaiyan, A.M., Rubin, M.A., Pienta, K.J., and Taichman, R.S., Expression of CXCR4 and CXCL12 (SDF-1) in human prostate cancers (PCa) in vivo. J Cell Biochem. 2003; 893:462-73.

38. Song, Z.Y., Wang, F., Cui, S.X., Gao, Z.H., and Qu, X.J., CXCR7/CXCR4 heterodimer-induced histone demethylation: a new mechanism of colorectal tumorigenesis. Oncogene. 2019; 389:1560-75.

39. Deng, L., Zheng, W., Dong, X., Liu, J., Zhu, C., Lu, D., Zhang, J., Song, L., Wang, Y., and Deng, D., Chemokine receptor CXCR7 is an independent prognostic biomarker in glioblastoma. Cancer Biomark. 2017; 201:1-6.

\section{Figures}

A

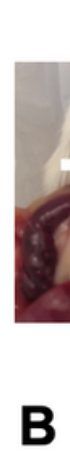

D28

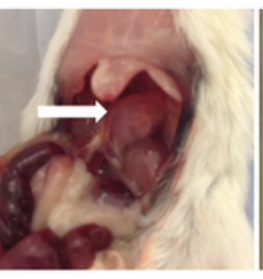

B
D49

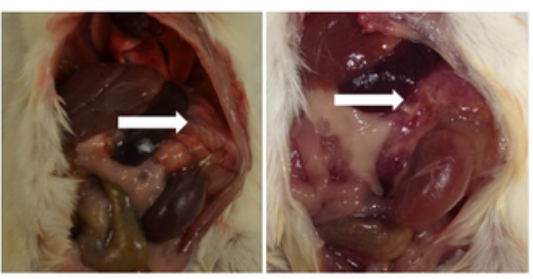

(i)

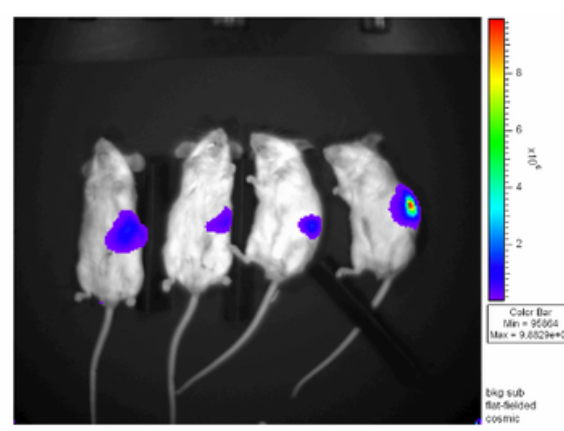

C

(i)

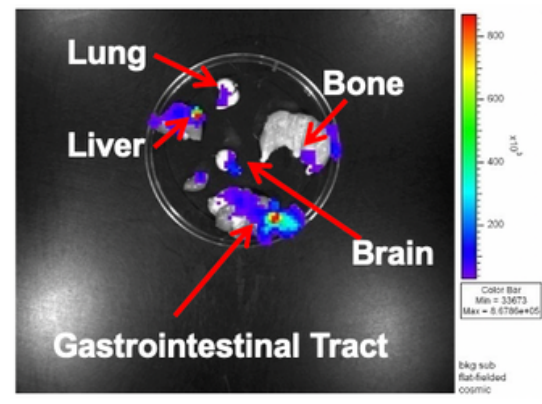

(ii)

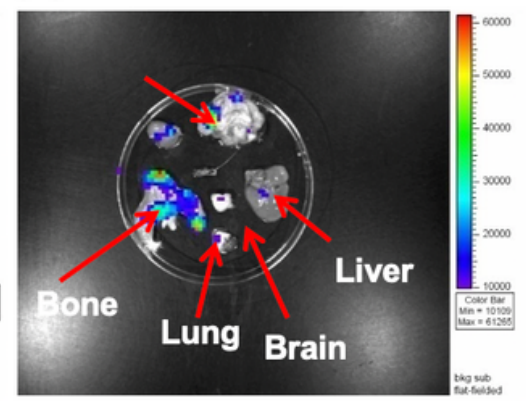

(ii)

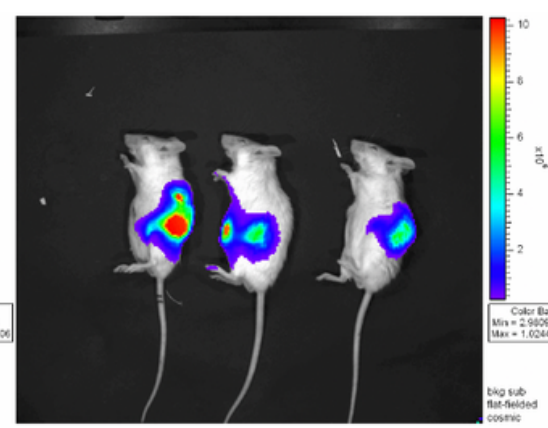

D

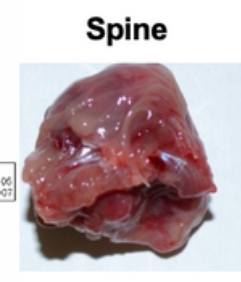

Liver \& GI Tract

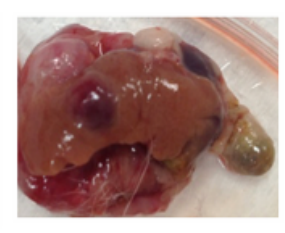

Bilateral Kidneys

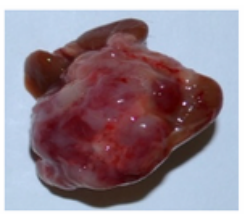

Figure 1 
Tumor growth and metastasis of orthotopic neuroblastoma model. Tumor developed at adrenal inoculation site at Day 28, Day 49 and Day 56 after SK-N-LP cell transplantation, which could be directly observed in mice (A). Bioluminescent detection of the tumor growth was demonstrated at Day $28(B(\mathrm{i}))$ and Day 56 (B(ii)) in vivo by IVIS in vivo imaging system, respectively (B). The color bar on right hand side showed the gradient of tumor activity from the highest (red) to the lowest (dark blue). Local and distant metastasis in brain, lung, liver, gut and bone was observed by detecting the bioluminescent signal (C(i)). Involvement of bone and spine as major organs rather than liver and lung was observed in some mice (C(ii)). Gastrointestinal tract, kidney, liver and lower spine encompassed by tumor could be directly observed (D).

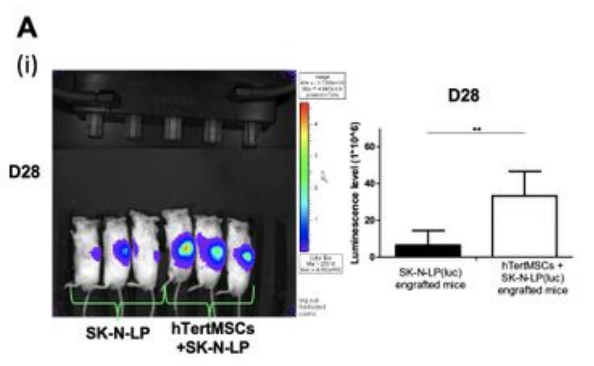

\section{B}
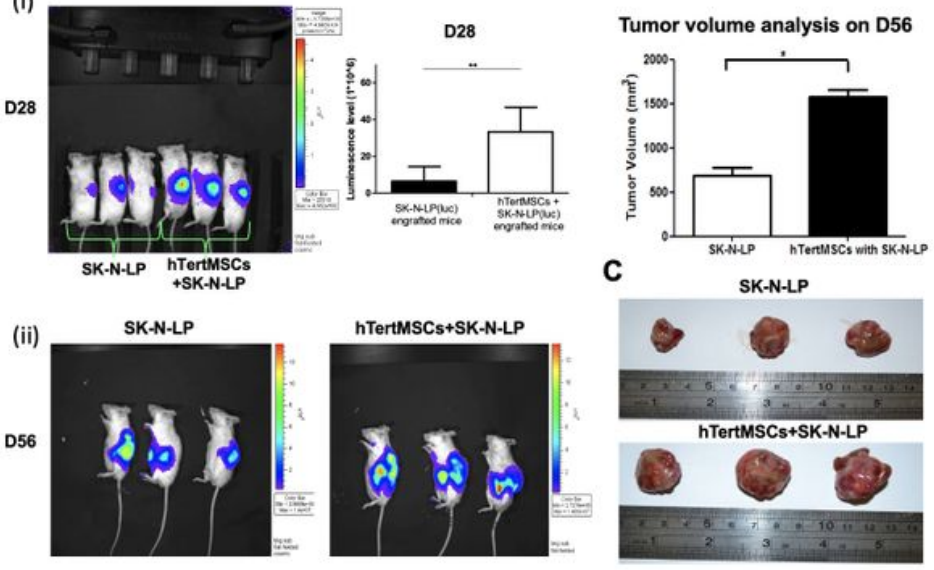

D

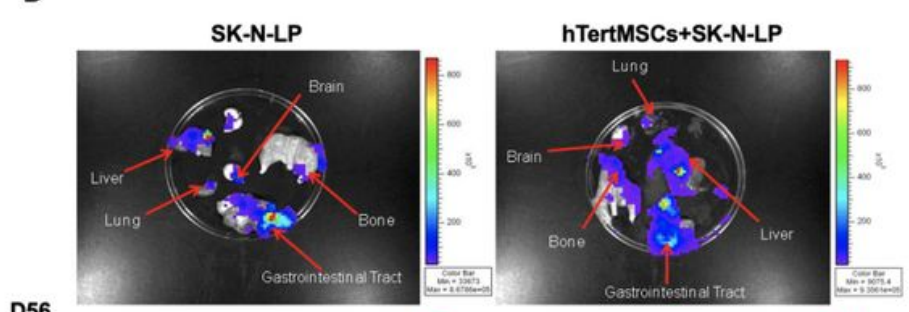

D56

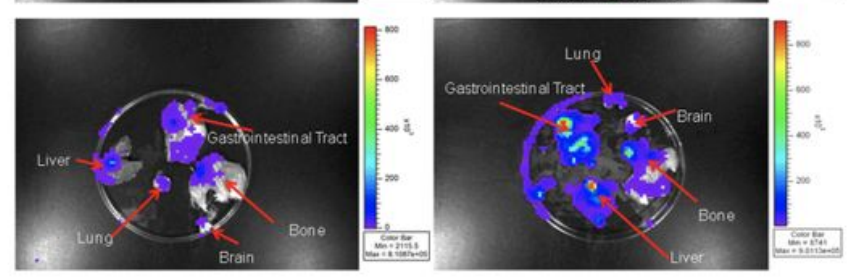

\section{Figure 2}

hMSCs enhanced tumor growth and metastasis. Significant difference in tumor bioluminescence could be observed at Day 28 between the group of co-transplanted with hMSCs and SK-N-LP alone group detected by Xenogen IVIS 100 in vivo imaging $(A(i))$. **indicates $P<0.01$. Such finding was more pronounced at Day 56 (A(ii)). Tumors were harvested (both groups) on Day 56 post-transplantation and the group with hMSCs and SK-N-LP co-transplantation showed significantly higher volume (B) and bigger tumor size (C) comparing to the SK-N-LP alone group. *indicates $\mathrm{P}<0.05$. Enhanced metastasis was observed in harvested organs (lung, liver, gastrointestinal tract, brain and bone) from co-transplantation group at Day 56 post-transplantation by detecting bioluminescent signal of SK-N-LP (D). 

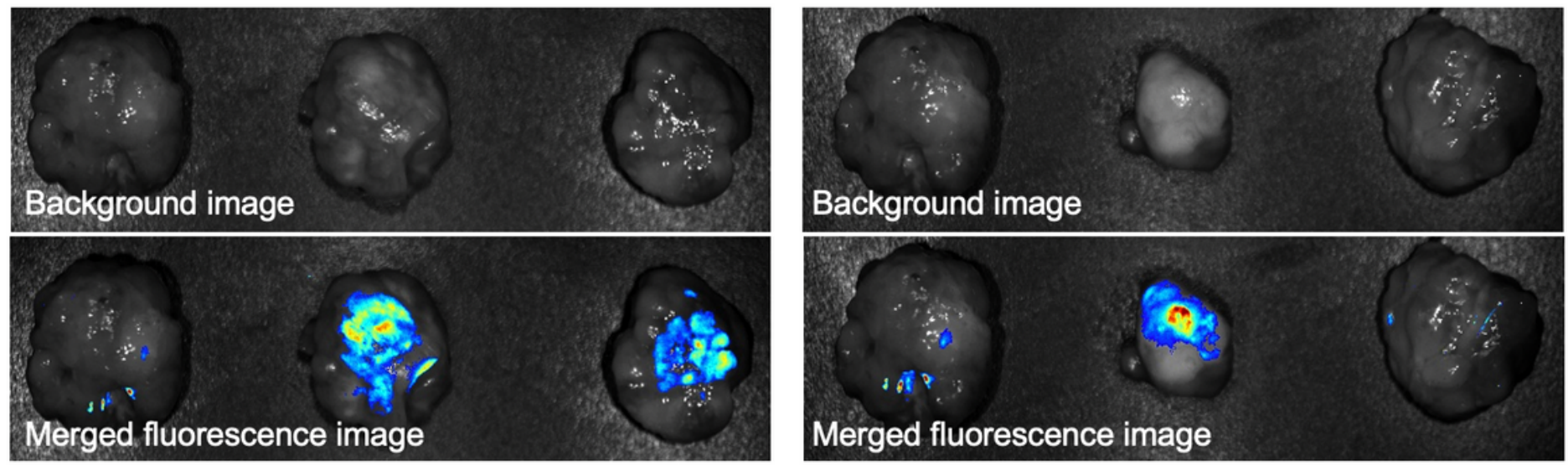

\section{Figure 3}

Recruitment of hMSCs towards primary tumor was CXCR4-dependent. CM-Dil-labeled hMSCs pre-treated with or without AMD3100, the specific CXCR4 antagonist, were injected into mice with neuroblastoma via tail vein. By detecting the fluorescence signal by CRI in vivo imaging system, hMSCs were observed to be preferentially attracted by primary tumor which was significantly inhibited by AMD3100.

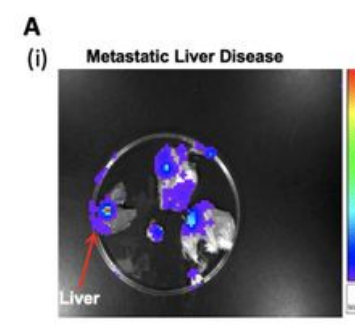

(ii) Liver without Metastatic Disease

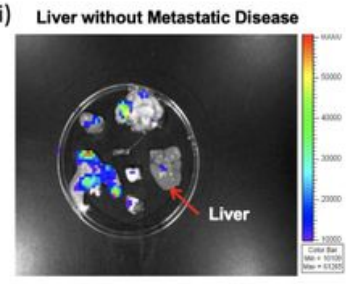

B

Normal Control Metastasis Non-metastasis

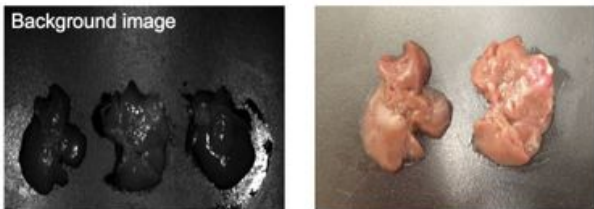

Fluorescence image

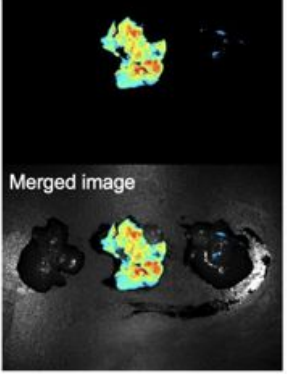

C

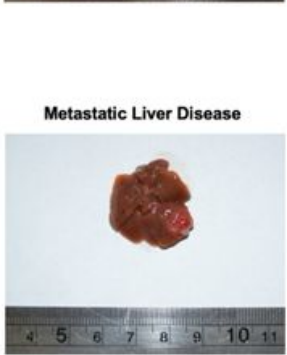

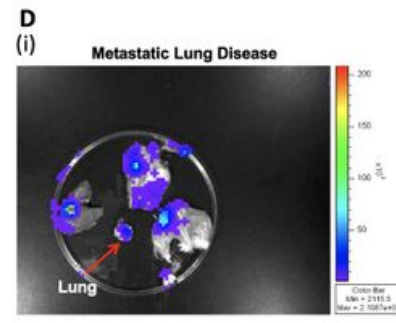

(ii)

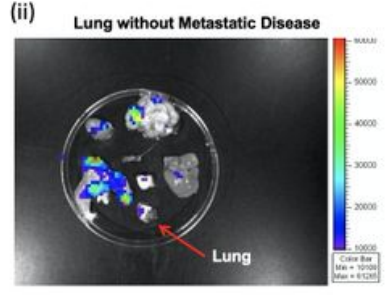

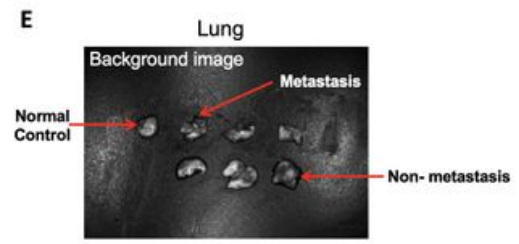
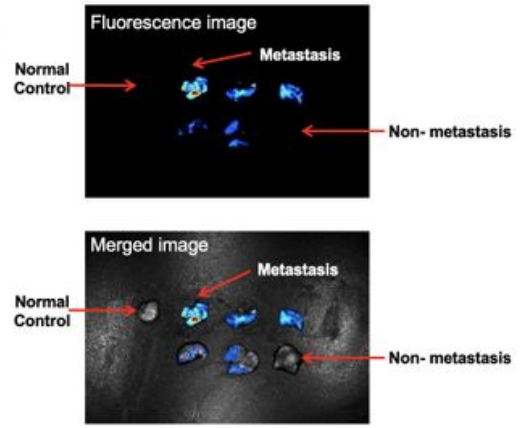

Figure 4

Selective recruitment of hMSCs by metastatic loci. The recruitment of hMSCs towards liver and lung with or without metastatic disease was compared, respectively and detected by fluorescence signal. The neuroblastoma metastasis in liver $(A(i))$ and lung $(D(i))$ was detectable by bio-luminescence using in vivo imaging system. No signal could be detected in liver ( $A($ ii) ) and lung $(D(i i))$ without metastatic disease. Liver metastatic loci could be directly observed in gross tumors ex vivo (C). hMSCs could be selectively attracted by liver (B) and lung (E) invaded by neuroblastoma cells. None could be detected in the normal liver (B) and lung (E). 

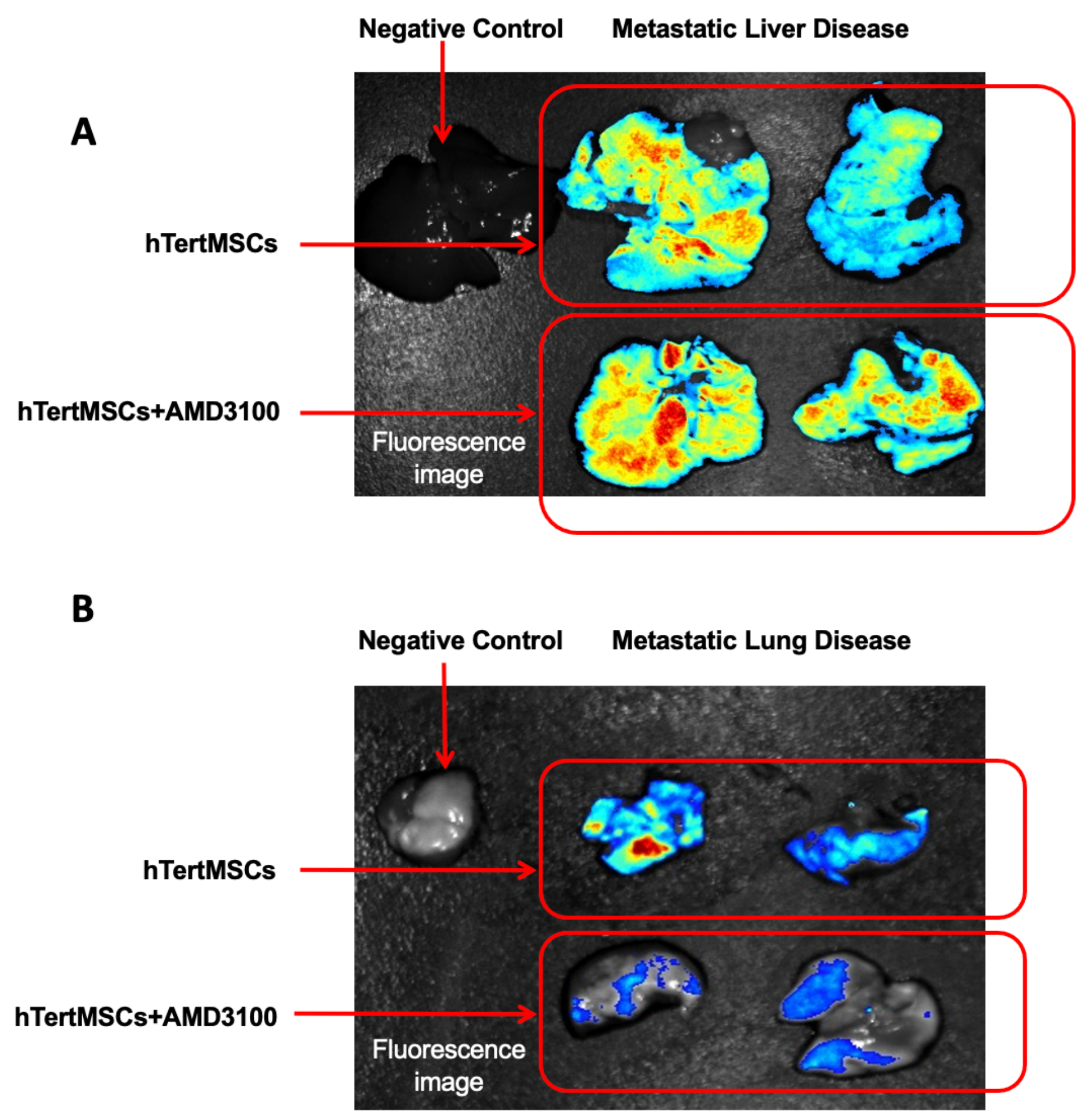

Figure 5

Recruitment of hMSCs by metastatic loci could not be completely inhibited by CXCR4 specific antagonist AMD3100. We traced the hMSCs trafficking in vivo by detecting the fluorescence signal of CM-Dil prelabeled hMSCs in freshly harvested livers and lungs. The treatment of AMD3100 could not completely inhibit the recruitment of hMSCs towards the liver with metastatic disease (A), and could partially block the recruitment towards the lung invaded with tumor cells (B). 

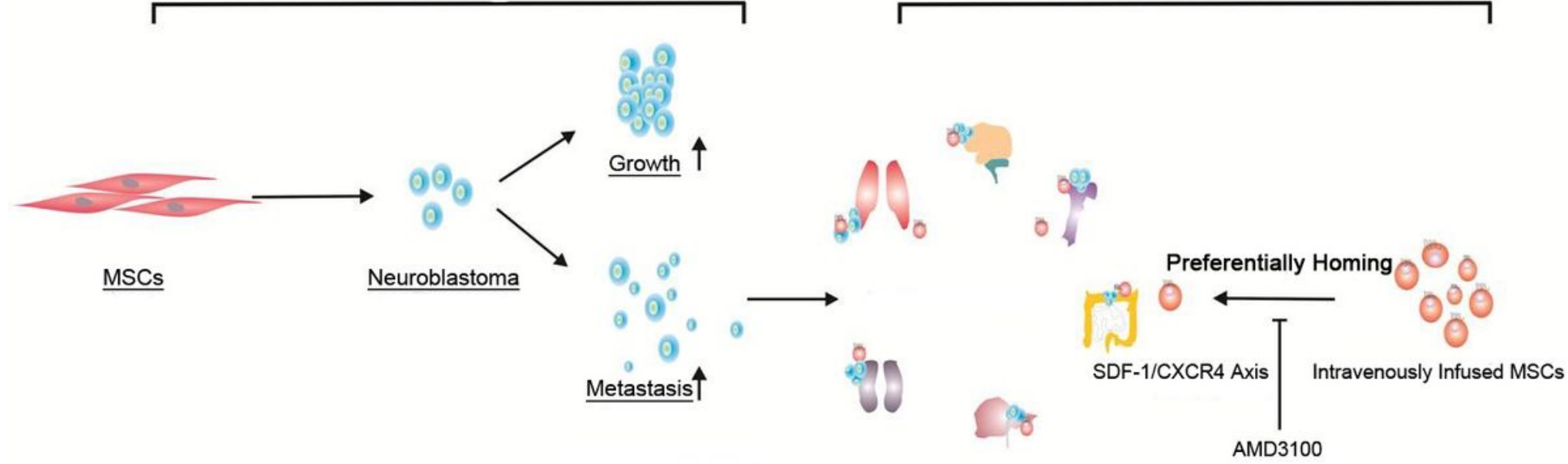

Orthotopic Mouse Model

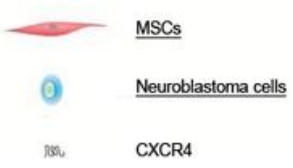

\section{Figure 6}

The "positive" and "anti-cancer" effects of MSCs on neuroblastoma. MSCs exhibited potent effects on accelerating the potency of tumor formation, growth and metastasis in vivo. On the other hand, intravenous infused MSCs could be recruited by both primary tumor and metastatic loci, which could be reduced when MSCs were pre-treated with SDF-1 blocker, AMD3100. The preferentially homing propensity of MSCs provides new therapeutic insights in utilizing bio-engineered MSCs as vehicles for targeted anticancer therapy against advanced cancers.

\section{Supplementary Files}

This is a list of supplementary files associated with this preprint. Click to download.

- SupplementaryFig1.ppt

- ARRIVEchecklist.docx 